\title{
EFEITOS DA UTILIZAÇÃO DE MISTURAS DE ADUBOS COM OU SEM ENXOFRE NA PRECOCIDADE E NAS CARACTERÍSTICAS DO CAPULHO E DA FIBRA DO ALGODOEIRO ( $\left.{ }^{1}\right)$
}

NELSON PAULIERI SABINO (2), Seção de Tecnologia de Fibras, e NELSON MACHADO DA SILVA, Seçāo de Algodāo, Instituto Agronômico.

\section{RESUMO}

São apresentados resultados referentes à precocidade e características do capulho e da fibra do algodoeiro, obtidos em ensaio de caráter permanente, no municipio de Guaíra (SP), em gleba de Latossolo Roxo, durante o periodo 1974/75-1977/78, utilizando-se a variedade 'IAC 16'. Além da reação ao fósforo, foi planejado um estudo conjunto visando observar a resposta do algodoeiro à aplicaçāo de misturas de adubo contendo fósforo e enxofre em quantidades variáveis. A análise e a interpretação dos resultados permitiram as seguintes conclusóes: a) Adubaçōes com superfosfato triplo ou simples, em solo deficiente em fósforo, resultaram em maior precocidade no ciclo do algodoeiro, enquanto o uso de sulfato de amônio em cobertura tendeu a prolongar esse ciclo; b) Ambas as fontes citadas de fósforo proporcionaram aumentos significativos no peso de capulho e no comprimento das fibras, enquanto apenas o superfosfato simples aumentou sensivelmente o peso de cem sementes e o índice Micronaire, que representa o complexo finura + maturidade da fibra; c) As características porcentagem de fibras, uniformidade de comprimento, resistência e maturidade das fibras, não foram alteradas significativamente pelos tratamentos estudados.

(1) Recebido para publicação a 30 de agosto de 1982.

(2) Com bolsa de suplementação do CNPq. 


\section{INTRODUÇÃO}

Muitos pesquisadores têm dirigido seus trabalhos no sentido de esclarecer os efeitos de $\mathrm{N}, \mathrm{P}$ e $\mathrm{K}$ sobre o desenvolvimento do algodoeiro e seus reflexos na produção e outros caracteres da planta, do capulho e da fibra $(1,4,7)$.

No presente trabalho, procurou-se estudar o comportamento do algodoeiro, diante de misturas de adubos contendo ou nāo enxofre, utilizando-se combinações de produtos comerciais comuns. Para tanto, instalou-se, durante quatro anos, ensaio de caráter permanente no município de Guaíra, em Latossolo Roxo.

\section{MATERIAL E MÉTODOS}

O ensaio foi instalado em gleba de Latossolo Roxo, durante o periodo 1974/75-1977/78, utilizando-se o cultivar 'IAC 16', de larga utilização, na época, pelos cotonicultores da região.

Além da reação ao fósforo, foi planejado um estudo conjunto visando observar a resposta do algodoeiro à aplicação de misturas de adubos contendo fósforo e enxofre em quantidades váriáveis. Os adubos minerais empregados, assim como as porcentagens dos diversos elementos, são apresentados a seguir: sulfato de amônio, $20 \%$ de $\mathrm{N}$ e $23 \%$ de $\mathrm{S}$; salitre-do-chile, $15 \%$ de $\mathrm{N}$; nitrato de amônio, $33,5 \%$ de $\mathrm{N}$; superfosfato simples, $20 \%$ de $\mathrm{P}_{2} \mathrm{O}_{5}, 25-28 \%$ de $\mathrm{CaO}$ e $12 \%$ de $\mathrm{S}$ e, superfosfato triplo, $45 \%$ de $\mathrm{P}_{2} \mathrm{O}_{5}$ e $14-16 \%$ de $\mathrm{CaO}$. Tanto o nitrogênio como $o$ potássio foram cedidos às plantas em doses fixas. O primeiro foi aplicado na base de $52,2 \mathrm{~kg} /$ hectare de $\mathrm{N}$, sendo parte no plantio ( $8 \mathrm{~kg}$ / hectare) e o restante em cobertura, após o desbaste. O potássio foi aplicado na base de $50 \mathrm{~kg} /$ hectare de $\mathrm{K}_{2} \mathrm{O}$ nos dois primeiros anos e de $75 \mathrm{~kg} /$ hectare a partir do terceiro ano, utilizando-se cloreto de potássio, no sulco de plantio.

Os seguintes tratamentos foram distribuídos no campo, segundo o esquema de quadrado latino $6 \times 6: 1$ - testemunha, sem fósforo (t); 2 - 50kg/hectare de $\mathrm{P}_{2} \mathrm{O}_{5}$, aplicados no plantio $(p) ; 3-100 \mathrm{~kg} /$ hectare de $\mathrm{P}_{2} \mathrm{O}_{5}$, aplicados no plantio $(2 \mathrm{p}) ; 4$ - $50 \mathrm{~kg} /$ hectare de $\mathrm{P}_{2} \mathrm{O}_{5}$ e $30 \mathrm{~kg} /$ hectare de S, aplicados no plantio $(\mathbf{p}+\mathbf{s}) ; 5-100 \mathrm{~kg} /$ hectare de $\mathrm{P}_{2} \mathrm{O}_{5}$ e $60 \mathrm{~kg} /$ hectare de $\mathrm{S}$, aplicados no plantio $(2 \mathrm{p}+2 \mathrm{~s}) ; 6-100 \mathrm{~kg} /$ hectare de $\mathrm{P}_{2} \mathrm{O}_{5}$ e $69,2 \mathrm{~kg} /$ hectare de $\mathrm{S}$ aplicados no plantio e $50,8 \mathrm{~kg} /$ hectare de $S$ em cobertura $(2 p+4 s)$.

Devido à alta acidez do solo, aplicou-se, em setembro de 1974 , cerca de 1,2t/hectare de calcário dolomítico, sendo adicionadas mais $2 \mathrm{t} /$ hectare em maio de 1977. 
As parcelas experimentais tiveram quatro linhas de $5 \mathrm{~m}$ de comprimento, com espaçamento de $1 \mathrm{~m}$, sendo deixadas em cada fileira, em média, 35 plantas distanciadas de aproximadamente $0,14 \mathrm{~m}$.

Por ocasião da colheita, realizada em duas etapas, foram coletadas, das duas linhas centrais de cada parcela, amostras de 40 capulhos, visando a estudos de características do capulho e da fibra.

Dados de precocidade foram obtidos através da relação percentuai entre o peso da primeira colheita e o total de produçăo de cada parcela. $O$ peso de um capulho e o peso de cem sementes corresponderam a determinações médias, dadas em grama. A porcentagem de fibra representa o valor médio da porcentagem ponderal da fibra, obtida após o beneficiamento das amostras. Os resultados de comprimento da fibra, em milímetro, correspondem aos valores médios do comprimento " $2,5 \%$ ", fornecido pelo Fibrógrafo. A uniformidade de comprimento em porcentagem representa a relação entre os comprimentos " $50 \%$ e 2,5\%" obtidos através do Fibrógrafo. O complexa finura + maturidade, determinado mediante o aparelho Micronaire, representa a proporção do fluxo de ar que atravessa uma amostra de fibra, com peso de 3,24 gramas e sob pressão constante. A resistência, dada em gramas por Tex $\left({ }^{*}\right)$, é determinada no aparelho Pressley, e representa a resistência à tração de um pequeno feixe de fibras paralelas, quando as pinças do aparelho estão separadas de 1/8". A maturidade da fibra, dada em porcentagem, corresponde ao índice médio fornecido pelo Fibrógrafo, segundo o método proposto por SABINO et alii (8).

A avaliação estatística dos resultados foi baseada em análises conjuntas envolvendo os quatro anos de estudo, tendo em vista o caráter permanente do ensaio. Para a comparação entre as médias dos tratamentos, foi utilizado o teste de Duncan, a 5\% de probabilidade.

\section{RESULTADOS E DISCUSSÃO}

No quadro 1, são encontrados os dados médios das características do capulho (porcentagem de fibra, peso de um capulho e peso de cem sementes) e da fibra (comprimento, uniformidade de comprimento, finura + maturidade, resistência e maturidade), bem como resultados respectivos de análises estatísticas, obtidos na análise conjunta dos dades referentes ao período 1974/75-1977/78.

A aplicação de fósforo na ausência de enxofre, estudada pelo efeito de doses variáveis de superfosfato triplo, aumentou de forma quadrática o peso de um capulho. Com o uso de misturas contendo enxofre, notou-se tendência de crescimento linear nos valores médios dessa carac-

(*) Tex: peso em gramas de 1.000 metros de fibra. 


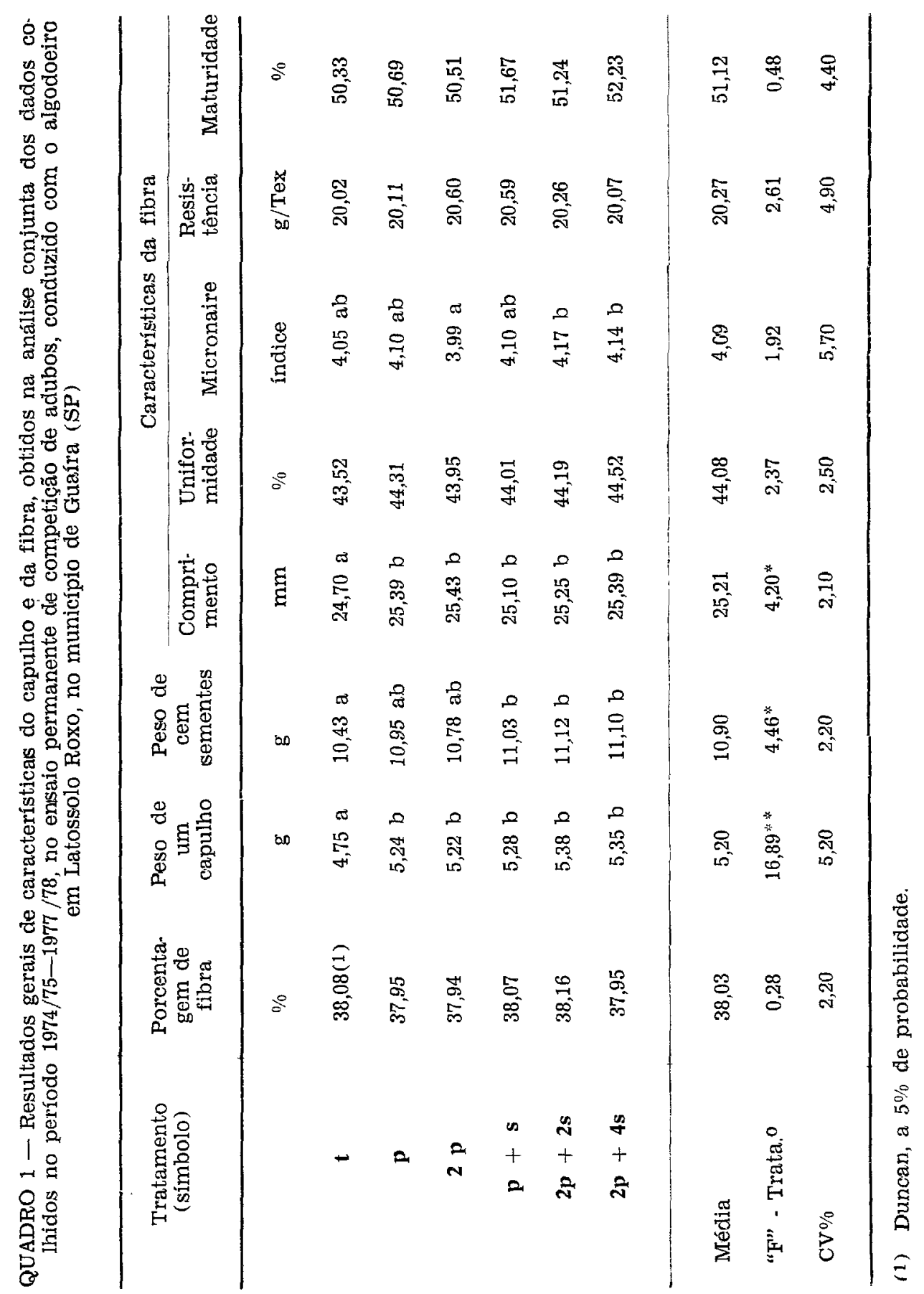




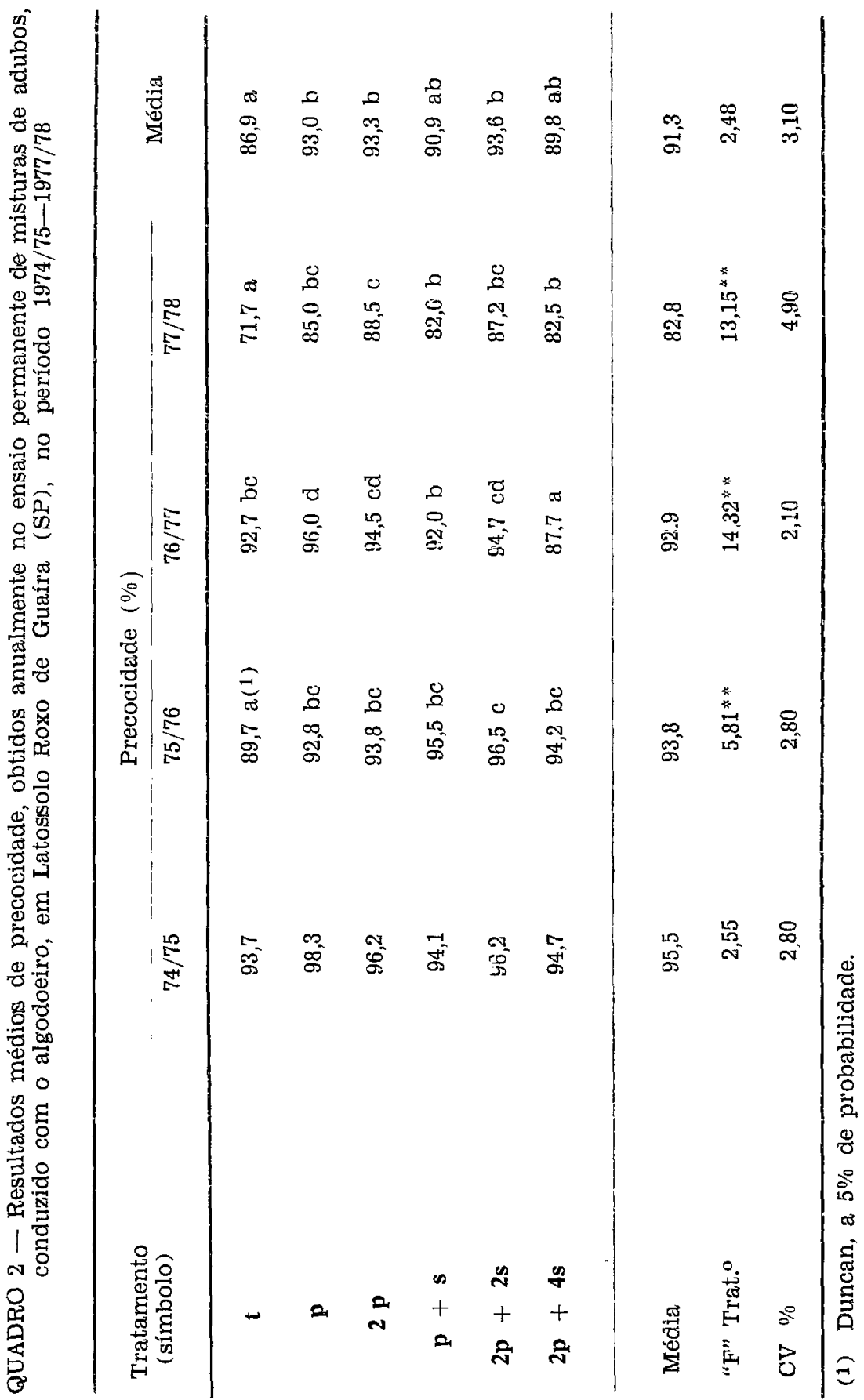


terística. Doses crescentes de $\mathrm{P}_{2} \mathrm{O}_{5}$, fornecidas pelo superfosfato triplo, não alteraram significativamente o peso de cem sementes, tendo sido observado, entretanto, efeito significativo dessas mesmas doses de $\mathrm{P}_{2} \mathrm{O}_{5}$, quando fornecidas pelo superfosfato simples.

De modo geral, pode-se afirmar que através do fornecimento associado de fósforo e enxofre às plantas, foram obtidos capulhos maiores com sementes mais pesadas.

Quanto às qualidades da fibra, o fósforo, na presença ou não de enxofre, beneficiou em especial o comprimento, o que vem confirmar resultados anteriores obtidos por diversos pesquisadores $(\mathbf{2}, \mathbf{5}, \mathbf{6})$.

As características porcentagem de fibra, uniformidade de comprimento, resistência e maturidade não foram influenciadas significativamente, quer por doses crescentes de fósforo, quer pelos tratamentos contendo enxofre.

Com respeito ao índice Micronaire, observou-se efeito positivo do enxofre na presença de dose máxima de fósforo, quando foram comparados os tratamentos de números $3(\mathbf{2 p})$ e $5(\mathbf{2 p}+\mathbf{2 s})$.

No quadro 2, são apresentados os resultados médios de precocidade das plantas, obtidos por tratamento e por ano, e os dados das respectivas análises das variâncias, durante o período 1974/75-1977/78. Para essa característica, a análise conjunta revelou um valor de "F" $=7,99^{* *}$ para a interação tratamentos $\mathrm{x}$ anos e, por esse motivo, optou-se pela apresentação dos dados obtidos anualmente.

No primeiro ano de condução ço ensaio, não foram observados efeitos da aplicação de adubos na precocidade das plantas, à semelhança do que ocorreu com os dados de produtividade (9). A partir do segundo ano-agrícola, o algodoeiro passou a responder mais à adubação, de modo geral. O efeito do fósforo desıacou-se a partir de então, concorrendo sempre para aumentar o nível relativo da primeira colheita, o que confirma o importante papel que exerce como regulador da maturação e da abertura dos frutos, conforme já indicado anteriormente (3). O uso de sulfato de amônio em cobertura, ao contrário concerreu para um relativu atraso no ciclo das plantas.

\section{CONCLUSÕES}

A análise e a interpretação dos dados relativos aos quatro anos em que o ensaio foi conduzido, permitiram as seguintes conclusões:

a) Adubações com superfosfato triplo ou simples, em solo deficiente em fósforo, resultaram em maior precocidade no ciclo do algodoeiro, enquanto o uso de sulfato de amônio em cobertura tendeu a prolongar esse ciclo; 
b) Ambas as fontes citadas de fósforo proporcionaram aumentos significativos no peso de capulho e no comprimento das fibras, enquanto apenas o superfosfato simples aumentou sensivelmente o peso de cem sementes e o índice Micronaire, que representa o complexo finura + maturidade da fibra;

c) As características porcentagem de fibras, uniformidade de comprimento, resistência e maturidade das fibras, não foram alteradas significativamente pelos tratamentos estudados.

\section{SUMMARY}

\section{EFFECTS OF MIXTURES OF FERTILIZERS WITH OR WITHOUT SULFUR ON COTTON EARLINESS AND SOME CHARACTERISTICS OF FIBERS AND BOLLS}

Effects of fertilizers mixturels with or without sulfur on cotton earliness and some characteristics of fiber and bolls, obtained in a field experiment carried out at Guaira County (SP) in a oxisoil - "Latossolo Roxo" - during the years of $1974 / 75$ to $1977 / 78$, are related. The following conclusions can be drawn from the results: a - soil fertilizations with concentrated superphosphate or ordinary superphosphate resulted in early picking of cotton crop. Plant cycle, was delayed with split application of ammonium sulphate; b - both concentrated superphosphate and ordinary superphosphate increased significantly boll weight and fiber lenght, but only ordinary superphosphate gave significant increases on seed weights and Micronaire values; $\mathrm{c}$ - length uniformity, maturity, strength and percentage of fiber were not influenced by these treatments.

\section{REFERENCIAS BIBLIOGRÁFICAS}

1. BENNET, O. L.; ROUSE, R. D.; ASHLEY, D. A.; DOSS, B. D. Yield, fiber quality and potassium content of irrigate cotton plants as affected by rates of potassium. Agronomy Journal, 57:296-299, 1965.

2. BROWN. H. B. A study of the effect of fertilizers on various characters of the cotton fiber. Louisiana, Agr. Exp. Sta., 1946. 47p. (Bulletin, 406)

3 FUZATTO, M. G. Adubação mineral. In: CULTURA e adubação do algogodoeiro. São Paulo, Instituto Brasileiro da Potassa, 1965. p.475-508.

4. HOOTEN, D. R.; JORDON, H. V.; PORTER, D.; JENKINS, P. M.; ADAMS, $J$. F. Influence of fertilizers on growth rates, fruiting habits, and fiber characters of cotton. Washington, Dept. Agriculture, 1949. 31p. (Technical bulletin, 779)

5. FOPE, O. A. Effects of certain soil types, seasonal conditions and fertilizer treatments on length and strength of cotton fiber. Arkansas, Agr. Exp. Sta., 1935. 98p. (Bulletin, 319)

6. REYNOLDS, E. B. \& KILLOUGH, D. T. The effect of fertilizers and rainfall on the length of cotton fiber. Journal American Society of Agronomy, 25: 756-764, 1963 . 
7. SABINO, N. P. Efeitos da aplicação de calcário, fósforo e potássio, na qualidade da fibra do algodoeiro cultivado em Latossolo Roxo. Bragantia, Campinas, 34(7):153-161, 1975.

8. - - GRIDI-PAPP, I. L.; KONDO, J. I.; CARNEIRO, J. B. Maturidade da fibra de algodão determinada pelo Fibrógrafo modelo 430. Bragantia, Campinas, 39(9):69-77, 1980 .

9. SILVA, N. M.; RODRIGUES FILHO, F. S. O. \& HIROCE, R. Uso de misturas de adubos contendo ou não enxofre na adubação do cultivar IAC 16 de algodoeiro. Bragantia, Campinas, 40:167-178, 1981. 